\title{
A Two Dimensional Discrete Fourier TRANSFORM BASED SECRET DATA EMBEDDING FOR COLOR IMAGE AUTHENTICATION (2D-DFTSDECIA)
}

\author{
J. K. Mandal ${ }^{1}$ and S. K.Ghosal ${ }^{2}$ \\ ${ }^{1}$ Department of Computer Science and Engineering, Kalyani University, Kalyani, \\ West Bengal, India \\ $j \mathrm{~km}$.cse@gmail.com \\ ${ }^{2}$ Department of Computer Science and Engineering, Greater Kolkata College of \\ Engineering \& Management, Baruipur, West Bengal, India \\ sudipta.ghosal@gmail.com
}

\begin{abstract}
In this paper, a novel two-dimensional Discrete Fourier Transform based information embedding has been proposed for color image authentication (2D-DFTSDECIA). Initially, each $2 \times 2$ sub-image block of the carrier image is pre-adjusted, if necessary and then transformed by the application of two dimensional Discrete Fourier Transforms (2D-DFT) where the transformation technique has been applied in row major order. In the proposed 2D-DFTSDECIA scheme, two bits from the authenticating message/image can be embedded in second, third and fourth frequency components of every $2 \times 2$ green sub-image matrix. Similarly, three bits from the authenticating message/image are embedded in second, third and fourth frequency components of every $2 \times 2$ red and blue sub-image matrices because, the human eye is less sensitive in blue and red channels as compared to the green channel. The first frequency component of green channel hides one bit at the pre-LSB position whereas two bits are embedded in first frequency component of red and blue channels starting from the pre-LSB. After embedding authenticating image/message bits, a frequency adjustment method is applied to get frequency component values closer to the actual frequency component value without hampering the embedded bits. Inverse $2 D$-DFT (2D-IDFT) is applied on each $2 \times 2$ image block after embedding authenticating message/image bits to generate embedded image. During the inverse transform phase, if any fractional value occurs in spatial domain, then the least significant bit of the first frequency component of that sub-matrix is flipped and 2D-IDFT is applied again. Experimental results conform that the proposed algorithm performs better than DPTHDI [7] and previously proposed DFTIECIA [8] schemes in terms of qualities and payload capacities.
\end{abstract}

\section{KEYWORDS}

2D-DFTSDECIA, DFTIECIA, DPTHDI, DFT, IDFT and pre-LSB.

\section{INTRODUCTION}

The concept of information hiding is to keep secret data safe from the intruders as the internet technology has become popular [1]. Steganography and invisible watermarking are two important fields of research which deals with the information hiding scheme. 2D-DFTSDECIA has been proposed for color images where the information embedding scheme has been used for the authentication purpose. Steganography can be broadly classified as operating in two different domains, such as spatial domain and frequency domain. 
Signal \& Image Processing : An International Journal (SIPIJ) Vol.3, No.6, December 2012

Frequency domain methods are widely used than spatial domain techniques to enhance the robustness as the secret data are embedded in both positive and negative frequency components. A number of works have been done for information hiding in this domain. Most common transformations are the Discrete Cosine Transformation (DCT), Quaternion Fourier Transformation (QFT), Discrete Fourier Transformation (DFT) and Discrete Wavelet Transformation (DWT). In transform domain, hidden data are embedded into the frequency component instead of spatial domain image pixel. I. J. Cox et al. [2, 3] developed an algorithm to inserts watermarks into the frequency components and spread over all the pixels. DCT-based image authentication is developed by N. Ahmidi et al. [4] using just noticeable difference profile [5] to determine maximum amount of watermark signal that can be tolerated at each region in the image without degrading visual quality. In [7], a new blind fragile data hiding technique has been proposed for secretly embedding messages into color images using the Discrete Pascal Transform (DPT) which has been proved remarkably useful for edge detection, filter design, discrete-time signal interpolation.

The Discrete Fourier Transformation is used to transform spatial domain image bytes to frequency components. The bits are embedded in each frequency component based upon the channel we have chosen. After embedding the secret data, Inverse Discrete Fourier Transformation is applied to get back the image in spatial domain.

So, the 2D-DFT of spatial domain pixel values $\mathrm{f}(\mathrm{x}, \mathrm{y})$ for the image of size $\mathrm{M} \times \mathrm{N}$ can be defined in eqn. (1) as:

$F(u, v)=\frac{1}{M N} \sum_{x=0}^{M-1} \sum_{y=0}^{N-1} f(x, y) e^{-j 2 \pi(u x / M+v y / N)}$

Where, $\mathrm{u}=0$ to $\mathrm{M}-1$ and $\mathrm{v}=0$ to $\mathrm{N}-1$.

The variable $\mathrm{u}$ and $\mathrm{v}$ are the transform or frequency variables and $\mathrm{x}, \mathrm{y}$ are the spatial or image variables and $f(x, y)$ are intensity values of pixels in spatial domain. Similarly, 2D-IDFT is used to convert frequency components to spatial domain values, and is defined in eqn. (2).

$f(x, y)=\sum_{u=0}^{M-1} \sum_{v=0}^{N-1} F(u, v) e^{j 2 \pi(u x / M+v y / N)}$

Where, $\mathrm{u}=0$ to $\mathrm{M}-1$ and $\mathrm{v}=0$ to $\mathrm{N}-1$.

The major aim of 2D-DFTSDECIA is to protect secret information, restrict unauthorized access and provide image authentication scheme by maintaining the integrity of the authenticating message/image. Thee authentication process can be achieved by embedding the secret data along with the message digest MD (which is generated from secret message/image) into the carrier image with a minimum change in visual pattern and improved security against visual attacks.

Problem motivation and formulation of transformation technique is given in section 2. The section 3 of the paper deals with the proposed technique. Results, comparison and analysis are given in section 4. Conclusions are drawn in section 5 and after that references are given. 
Signal \& Image Processing : An International Journal (SIPIJ) Vol.3, No.6, December 2012

\section{TRANSFORMATION TECHNIQUES}

In this technique an image sub block of size $2 \times 2$ is taken and DFT is applied. The formulation of a 2 x 2 mask for four different image bytes in 2D-DFT is as follows:

$$
\begin{aligned}
& F\left(a_{0,0}\right)=1 / 2 \sum_{i=0}^{1} \sum_{j=0}^{l} a_{i, j}=c_{0,0} \text { (say), } \\
& F\left(a_{0,1}\right)=1 / 2 \sum_{i=0}^{1} \sum_{j=0}^{1}(-1)^{j} a_{i, j}=c_{0,1} \text { (say), } \\
& F\left(a_{1,0}\right)=1 / 2 \sum_{i=0}^{1} \sum_{j=0}^{1}(-1)^{i} a_{i, j}=c_{1,0} \text { (say), } \\
& F\left(a_{1,1}\right)=1 / 2 \sum_{i=0}^{1} \sum_{j=0}^{1}(-1)^{i}(-1)^{j} a_{i, j}=c_{1,1} \text { (say), }
\end{aligned}
$$

Where $\mathrm{c}_{0,0}, \mathrm{c}_{0,1}, \mathrm{c}_{1,0}$ and $\mathrm{c}_{1,1}$ are all frequency components for $\mathrm{a}_{0,0}, \mathrm{a}_{0,1}, \mathrm{a}_{1,0}$ and $\mathrm{a}_{1,1}$ spatial domain values respectively. Embedding is done up to the least three significant bits of $\mathrm{c}_{0,0}, \mathrm{c}_{0,1}, \mathrm{c}_{1,0}$ and $\mathrm{c}_{1,1}$ based upon the channel we have chosen. The LSB-0 of first frequency component $\left(\mathrm{c}_{0,0}\right)$ does not take part in embedding as the bit can be used to handle fractional pixel values during the inverse transform phase.

Similarly, by applying the inverse 2D-DFT, the $2 \times 2$ transformed masks can be converted back to spatial domain image bytes as follows:

$$
\begin{aligned}
& F\left(c_{0,0}\right)=1 / 2 \sum_{i=0}^{1} \sum_{j=0}^{l} c_{i, j}=a_{0,0} \text { (say), } \\
& F\left(c_{0,1}\right)=1 / 2 \sum_{i=0}^{1} \sum_{j=0}^{l}(-1)^{j} c_{i, j}=a_{0,1} \text { (say), } \\
& F\left(c_{1,0}\right)=1 / 2 \sum_{i=0}^{1} \sum_{j=0}^{1}(-1)^{i} c_{i, j}=a_{1,0}(\text { say }), \\
& F\left(c_{1,1}\right)=1 / 2 \sum_{i=0}^{l} \sum_{j=0}^{l}(-1)^{i}(-1)^{j} c_{i, j}=a_{1,1} \text { (say), }
\end{aligned}
$$

\section{Proposed Technique}

In this paper a new information embedding approach has been proposed for color image authentication (2D-DFTSDECIA) in frequency domain based on the two dimensional Discrete Fourier Transform (2D-DFT). Initially, a 128 bit message digests (MD) and sizes of the hidden data are embedded using the proposed 2D-DFTSDECIA scheme for authentication purpose. Each $2 \times 2$ sub-image block of the carrier image is pre-adjusted, if necessary. This pre-embedding pixel adjustment strategy ensures that the pixel values after embedding authenticating data in frequency domain has to be non-negative and not to be out of range $(0 \leq \mathrm{P} \leq 255)$. The 2D-DFT is applied on $2 \times 2$ sub-image block for converting the spatial domain pixel values to frequency components. This process is continued till the last sub-image block of the carrier/cover image in a row major order. The authenticating message/image is embedded in all the frequency components in each $2 \times 2$ sub-image matrix of the carrier image. In the proposed 2D-DFTSDECIA scheme, two bits from the authenticating message/image can be embedded in second, third and fourth frequency components of every $2 \times 2$ green sub-image matrix. Similarly, three bits from the 
Signal \& Image Processing : An International Journal (SIPIJ) Vol.3, No.6, December 2012

authenticating message/image are embedded in second, third and fourth frequency components of every $2 \times 2$ red and blue sub-image matrices. The green sub-matrix hide less bits from the authenticating message/image as compared to the red and blue sub-matrices as the human eye is most sensitive in green channel than red and blue channels. The first frequency component of green channel hides one bit at the pre-LSB position i.e. LSB-1, whereas two bits are embedded in first frequency component of red and blue channels starting from the pre-LSB. That means, for each red and blue sub-matrix can hides eleven bits whereas each green sub-matrix can hide seven bits from the authenticating message/image. The least significant bits (LSB-0) of the first frequency component has not been used for embedding purpose because it has to be used for readjustment of frequency components whenever it produce a fractional pixel value representation. In the proposed technique, if the value of frequency components becomes fractional, then the LSB-0 of the first frequency component is flipped. After embedding authenticating bits, a postembedding adjustment of the frequency components has been applied to ensure the frequency components value closest to the original frequency components without hampering the hidden bits. A two dimensional inverse Discrete Fourier Transforms (2D-IDFT) has been applied to get back the less distorted embedded image in spatial domain. In the reverse manner, we can extract the authenticating data from the embedded image and new message digest MD' can be calculated from the extracted authenticating bits and the same is compared with extracted MD for authentication.

For example, let we have chosen the Baboon image as the cover/carrier image. At first, we have applied 2D-DFT on the first $2 \times 2$ sub-image block to convert it from spatial domain pixel values to frequency components value in transform domain where $\mathrm{R} 1, \mathrm{G}_{1}$ and $\mathrm{B}_{1}$ are the sub-matrices for the first 2 x 2 sub-image block. The steps are as shown below:

$R_{l}=\{164,63,120,135\}, G_{l}=\{150,57,125,97\}, B_{1}=\{71,31,62,33\}$

After, applying 2D-DFT we get the transformed frequency component values as shown below:

$F\left(R_{1}\right)=\{241,43,-14,58\}, F\left(G_{1}\right)=\{214,60,-7,32\}, F\left(B_{1}\right)=\{98,34,3,5\}$

Now, if we embed authenticating bits having the binary representation of 01000001011110101100101000001 using the proposed scheme, then the embedded frequency components for the first $2 \times 2$ mask becomes:

$E F\left(R_{1}\right)=\{243,40,-9,59\}, E F\left(G_{1}\right)=\{214,62,-6,35\}, E F\left(B_{1}\right)=\{98,37,0,1\}$

The frequency adjustment method ensures that the bits which are not taking part in embedding can choose different combinations of 0 's and 1's. Consequently, the value closest to the embedded frequency component is chosen without hampering the hidden authenticating bits.

$A\left(E F\left(R_{1}\right)\right)=\{243,40,-17,59\}, A\left(E F\left(G_{1}\right)\right)=\{214,60,-7,35\}, A\left(E F\left(B_{1}\right)\right)=\{98,37,0,1\}$

Again, applying 2D-IDFT we get back the values in pixel domain. During this 2D-IDFT phase, we can see that the pixel values in red matrix are fractional. Thus, to make the pixels nonfractional, the LSB-0 of first frequency component bit is flipped and 2D-IDFT is applied again. As a result, the embedded sub-matrices becomes as follows:

$F^{-1}\left(A\left(E F\left(R_{1}\right)\right)\right)=\{162,63,120,139\}, F^{-1}\left(A\left(E F\left(G_{1}\right)\right)\right)=\{151,56,123,98\}$,

$F^{-1}\left(A\left(E F\left(B_{1}\right)\right)\right)=\{68,30,67,31\}$ 
Signal \& Image Processing : An International Journal (SIPIJ) Vol.3, No.6, December 2012

Actually, the process of embedding is continued for each $2 \times 2$ mask till the last hidden bit. We have categorized this section into two parts namely the Algorithm for Insertion and the Algorithm for Extraction.

\subsection{Insertion}

All insertion is made in frequency domain i.e. each byte of source image in each mask of size $2 \times$ 2 is transformed to frequency domain using two dimensional DFT equations. The proposed scheme uses color image as the input to be authenticated by text message/image. All the three channels in a 24 bit color image have been chosen for embedding purpose. As we know, the perceptibility of green channel is comparatively high than red and blue channel, for that purpose our intention is to embed less bit in green channel as compared to the other two channels, so, our tendency is to embed more bits in red and blue channels. The authenticating message/image bits size is $\beta \times \mu \times(m \times n)-(M D+L)$ where $\beta$ is the average bit per byte, $\mu$ is the total number of channels in the cover image which is 3 for color image, MD and L are the message digest and dimension of the authenticating image respectively for the source image of size $\mathrm{m} \times \mathrm{n}$ bytes. The value of $(\mathrm{MD}+\mathrm{L})$ is always to be less than $\beta \times \mu \times(\mathrm{m} \times \mathrm{n})$.

\section{Steps:}

1) Obtain $r$ bits message digest $M D$ from the authenticating message/image.

2) Obtain the size of the authenticating message/image $((L=W+H)$ bits, where $W$ bits for width and $H$ bits for height). The authenticating message/image bits size is:

$$
\beta \times \mu \times(M \times N)-(M D+L)
$$

Where, $M D$ and $L$ are the message digest and dimension of the authenticating image respectively for the source image size of $M$ x $N$ bytes. In our proposed scheme, $\beta$ is 2.41 and $\mu$ is 3 , whereas the $M D$ and $L$ consist of 128 and 32 bits.

3) Read authenticating message/image data do:

- The cover image is partitioned into $2 \times 2$ non-overlapping blocks in row major order. Each $2 \times 2$ block is consists of four pixels, $p_{i, j}, p_{i, j+1}, p_{i+1, j}$ and $p_{i+1, j+1}$ where the values of $i$ and $j$ lies in the range $0 \leq i \leq l$ and $0 \leq j \leq 1$.

- Reset the upper limit and lower limit of pixel component value $\left(p_{c}\right)$ to retain the spatial domain pixel component values positive and less than, or equal to 255 during the inverse transformation at later step. The initial pixel adjustment has been made on the basis of the concept that human eye is less sensitive in blue and red channels as compared to the green channel. That means,

$$
\begin{aligned}
p_{c} & =\left\{240 ; \text { if }\left(p_{c} \geq 240\right) \text { and }(c=R \text { or } B)\right. \\
& =\mid 16 ; \text { if } p_{c} \leq 16 \text { and }(c=R \text { or } B) \\
& =\mid 248 ; \text { if }\left(p_{c} \geq 248\right) \text { and }(c=G) \\
& =\mid 8 ; \text { if } p_{c} \leq 8 \text { and }(c=G)
\end{aligned}
$$


Signal \& Image Processing : An International Journal (SIPIJ) Vol.3, No.6, December 2012

- Apply two dimensional DFT on each sub-matrices corresponding to the red, green and blue channels separately which produces four frequency components $f_{i, j}, f_{i, j+1}, f_{i+1, j}$ and $f_{i+1, j+1}$ with respect to each sub-matrix.

- $\mathrm{N}$ bits are embedded in 2nd, 3rd and 4th frequency component of each 2 x 2 mask starting from the LSB-0 and (N-1) bits are embedded in $1^{\text {st }}$ frequency component starting from the LSB-1 depending upon the channel $(\mathrm{R} / \mathrm{G} / \mathrm{B})$ we have chosen. The mathematical expression can be written as follows:

$$
\begin{aligned}
N & =\{3 ; \text { if }(c=R \text { or } B) \\
& =\mid 2 ; \text { if }(c=G)
\end{aligned}
$$

[Embed authenticating message/image bit as per the above rules.]

4) A delicate frequency adjustment method has been applied to get frequency components values closest to the original without hampering the hidden bits. The adjustment has been made by altering left most $(T-N)$ number of bits followed by choosing the specific frequency component value closest to the original one where $T$ is the total number of bits in a frequency component.

5) Apply two dimensional inverse DFT using identical masks. During the 2D-IDFT phase, if the pixel value in spatial domain becomes fractional; then the LSB-0 of the $1^{\text {st }}$ frequency component has to be flipped and then 2D-IDFT is applied again.

6) Repeat step 2 to step 5 for the whole authenticating message/image size, content and for message digest $M D$. The successive block embedding operation produces the embedded image $\left(I^{\prime}\right)$.

7) Stop.

\subsection{Extraction}

The authenticated image is received in spatial domain. During extraction the embedded image has been taken as the input and the authenticating message/image size, image content and message digest MD are extracted from it. All extraction is done in frequency domain from frequency components.

\section{Steps:}

1) The embedded image is partitioned into $2 \times 2$ non-overlapping blocks in row major order. Each $2 \times 2$ block is consists of four pixels, $p_{i, j}, p_{i, j+1}, p_{i+1, j}$ and $p_{i+1, j+1}$, where the values of $i$ and $j$ lies in the range $0 \leq i \leq l$ and $0 \leq j \leq 1$.

2) For each $2 \times 2$ mask do the following operations:

- To transform every $2 \times 2$ pixel blocks consisting of $p_{i, j}, p_{i, j+l}, p_{i+1, j}$ and $p_{i+1, j+l}$ from spatial domain to frequency domain, two-dimensional Discrete Fourier Transform (2D-DFT) has been applied on each sub-matrices corresponding to the red, green and blue channels separately which produces four frequency components $f_{i, j}, f_{i, j+1}, f_{i+1, j}$ and $f_{i+1, j+1}$, where the values of $i$ and $j$ lies in the range $0 \leq i \leq 1$ and $0 \leq j \leq 1$.

- $\quad \mathrm{N}$ bits are extracted from 2nd, 3rd and 4th frequency component of each $2 \times 2$ mask starting from the LSB- 0 and $(\mathrm{N}-1)$ bits are extracted from $1^{\text {st }}$ frequency component 
Signal \& Image Processing : An International Journal (SIPIJ) Vol.3, No.6, December 2012

starting from the LSB-1 depending upon the channel $(\mathrm{R} / \mathrm{G} / \mathrm{B})$ we have chosen. The mathematical expression can be written as follows:

$$
\begin{aligned}
N & =\{3 ; \text { if }(c=R \text { or } B) \\
& =\mid 2 ; \text { if }(c=G)
\end{aligned}
$$

[Extract authenticating message/image bit as per the above rules.]

- For each 8 (eight) bits extraction, construct one alphabet/one primary (R/G/B) color component.

3) Repeat step 1 and step 2 to complete decoding as per size of the authenticating message/image.

4) Obtain 128 bits message digest $\mathrm{MD}^{\prime}$ from the extracted authenticating message/image. Compare MD' with extracted MD. If both are same, then image is authorized, else, unauthorized.

5) Apply two dimensional inverse DFT using identical masks.

6) Stop

\section{ReSUlts, COMPARISON AND ANALYSIS}

This section represents the results, discussion and a comparative study of the proposed 2DDFTSDECIA technique with the DPTHDI and DFTIECIA methods in terms of visual interpretation, payload capacities, peak signal to noise ratio (PSNR) analysis and histogram analysis. Benchmark (PPM) images [6] are taken to formulate results and are shown in Fig-1. All cover images are 512 × 512 in dimension whereas the gold coin (i.e. the authenticating image) is embedded into the various source benchmark images. The performance of the proposed approach has been evaluated on ten different color images (i-x), where each pixel is represented by three intensity values RGB (Red, Green and Blue). Images are labelled as: (i) Lena, (ii) Baboon, (iii) Pepper, (iv) Airplane, (v) House, (vi) Earth, (vii) Sailboat, (viii) Foster City, (ix) San Diego, (x) Oakland. On embedding the Gold-Coin image of 237538 bytes, the embedded image produces a good visual clarity.

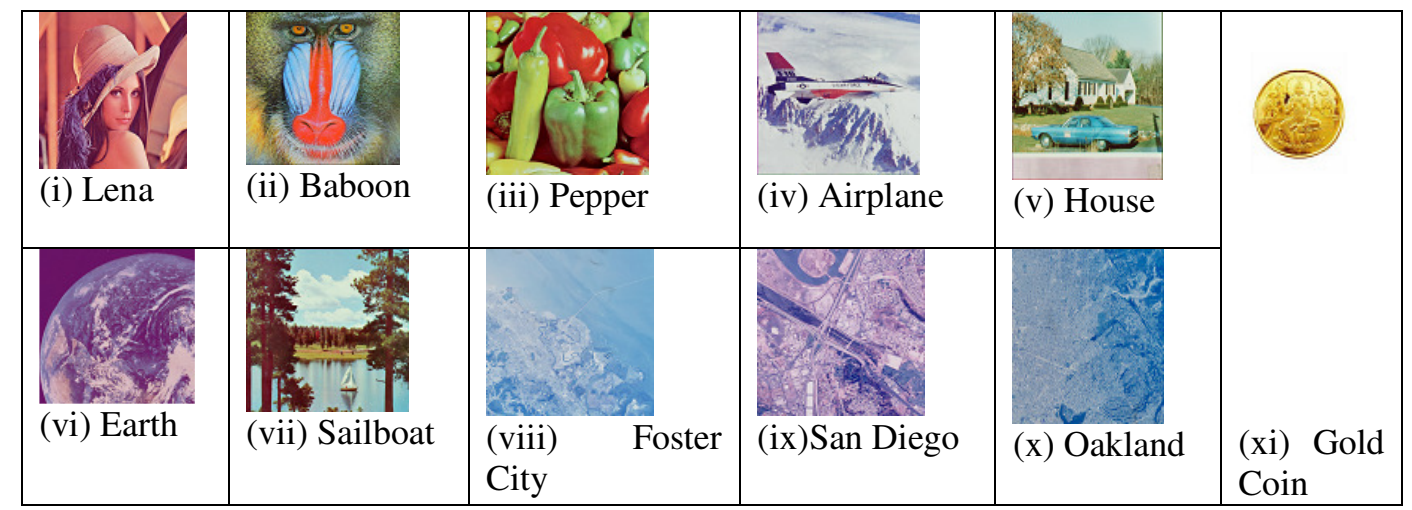

Figure 1. Different Cover images (512 x 512) and that of common authenticating image $(282 \times 280)$ 
Signal \& Image Processing : An International Journal (SIPIJ) Vol.3, No.6, December 2012

Based on the proposed technique and experimental results, we can see that the technique can overcome many kinds of visual and statistical attacks and it is quite difficult for the observer to detect the difference between the original and embedded image. From Table 1, we can identify the capacities for carrier images 'Lena', 'Baboon' and 'Pepper' are 237538 bytes where the dimension of each original image is 512 x 512. After, embedding a large amount of authenticating data the embedded images are also pertain a good visual clarity and produces value of around 38 $\mathrm{dB}$ for peak to signal noise ratio and 0.9993 for image fidelity in average case. Moreover, the histogram analysis shows the changes made in the 'Lena' image are more stable after embedding authenticating bits. The table also shows that the average number of bits embedded per byte (bpb) for ten images is 2.41. Fig-2 shows different states of modifications (before and after) of three different images viz. Lena, Baboon and Peppers.

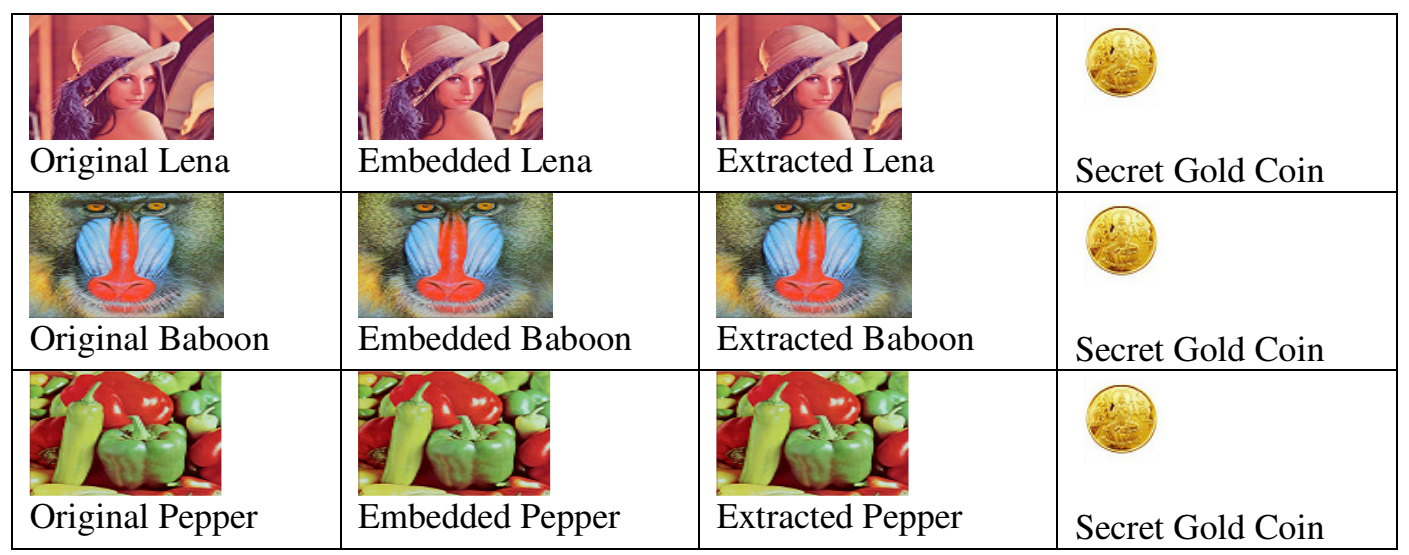

Figure 2. Cover, Embedded, Extracted and Secret Images using proposed 2D-DFTSDECIA scheme

Also, a comparative study has been made among the proposed 2D-DFTSDECIA technique with the DPTHDI and DFTIECIA methods based on capacities (bytes) and the PSNR values. In the proposed scheme, the capacity (bytes) is much more as compared to the DPTHDI and DFTIECIA technique, whereas the PSNR values is also enhanced and produces good visual clarity embedded images.

Table 1. Results of embedding 237538 bytes of information in each image of dimension $512 \times 512$

\begin{tabular}{|c|c|c|c|c|}
\hline Carrier Image & $\begin{array}{c}\text { Capacity } \\
\text { (byte) }\end{array}$ & $\begin{array}{c}\text { PSNR } \\
\text { (dB) }\end{array}$ & IF & $\begin{array}{c}\text { Bits Per Byte } \\
\text { (bpb) }\end{array}$ \\
\hline Lena & 237538 & 37.79 & 0.9994 & 2.41 \\
\hline Baboon & 237538 & 38.54 & 0.9995 & 2.41 \\
\hline Pepper & 237538 & 35.45 & 0.9979 & 2.41 \\
\hline Earth & 237538 & 38.32 & 0.9995 & 2.41 \\
\hline Sailboat & 237538 & 38.10 & 0.9995 & 2.41 \\
\hline Airplane & 237538 & 37.41 & 0.9997 & 2.41 \\
\hline Foster City & 237538 & 38.12 & 0.9996 & 2.41 \\
\hline Oakland & 237538 & 36.79 & 0.9992 & 2.41 \\
\hline San Diego & 237538 & 38.86 & 0.9997 & 2.41 \\
\hline House & 237538 & 37.32 & 0.9995 & 2.41 \\
\hline $\boldsymbol{A V G}$ & $\mathbf{2 3 7 5 3 8}$ & $\mathbf{3 7 . 4 7}$ & $\mathbf{0 . 9 9 9 3}$ & $\mathbf{2 . 4 1}$ \\
\hline
\end{tabular}


Signal \& Image Processing : An International Journal (SIPIJ) Vol.3, No.6, December 2012

Table 2. Comparison results of Capacities and Qualities in terms of bits per byte (bpb) and PSNR for four different images with the existing techniques namely DPTHDI and DFTIECIA

\begin{tabular}{|c|c|c|c|c|c|c|}
\hline Carrier Image & \multicolumn{2}{|c|}{ DPTHDI [7] } & \multicolumn{2}{c|}{ DFTIECIA [8] } & \multicolumn{2}{c|}{ 2D-DFTSDECIA } \\
\cline { 2 - 7 } & $\begin{array}{c}\text { BPB } \\
\text { (bits) }\end{array}$ & $\begin{array}{c}\text { PSNR } \\
\text { (dB) }\end{array}$ & $\begin{array}{c}\text { BPB } \\
\text { (bits) }\end{array}$ & $\begin{array}{c}\text { PSNR } \\
\text { (dB) }\end{array}$ & $\begin{array}{c}\text { BPB } \\
\text { (bits) }\end{array}$ & $\begin{array}{c}\text { PSNR } \\
\text { (dB) }\end{array}$ \\
\hline Lena & 2.25 & 39.70 & 2.39 & 31.96 & 2.41 & 37.79 \\
\hline Baboon & 2.25 & 30.69 & 2.16 & 34.08 & 2.41 & 38.54 \\
\hline Pepper & 2.25 & 37.40 & 2.42 & 31.89 & 2.41 & 35.45 \\
\hline Sailboat & 2.25 & 35.28 & 2.34 & 33.12 & 2.41 & 38.10 \\
\hline $\boldsymbol{A} \boldsymbol{V G}$ & $\mathbf{2 . 2 5}$ & $\mathbf{3 5 . 7 6}$ & $\mathbf{2 . 3 2}$ & $\mathbf{3 2 . 7 6}$ & $\mathbf{2 . 4 1}$ & $\mathbf{3 7 . 4 7}$ \\
\hline
\end{tabular}

In Fig. 3 and Fig. 4, the comparison result of the bpb and PSNR values for embedded 'Lena' image is shown in the form of a chart with varying sizes of carrier images viz. 64 x 64, $128 \times 128$, $256 \times 256$ and $512 \times 512$, where the comparison has been made among proposed 2DDFTSDECIA scheme with two existing schemes [7-8] previously mentioned by us. The chart also explains how efficiently we have enhanced the payload capacities along with the PSNR values.

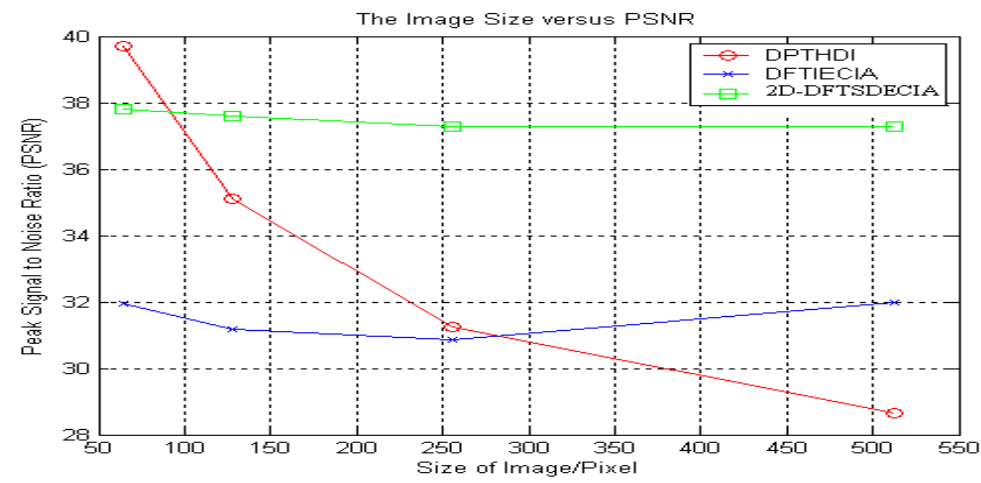

Figure 3. Comparisons Results of Size of Image/Pixel and PSNR among Varying Sizes of Embedded 'Lena' Image with schemes DPTHDI [7], DFTIECIA [8] and 2D-DFTSDECIA

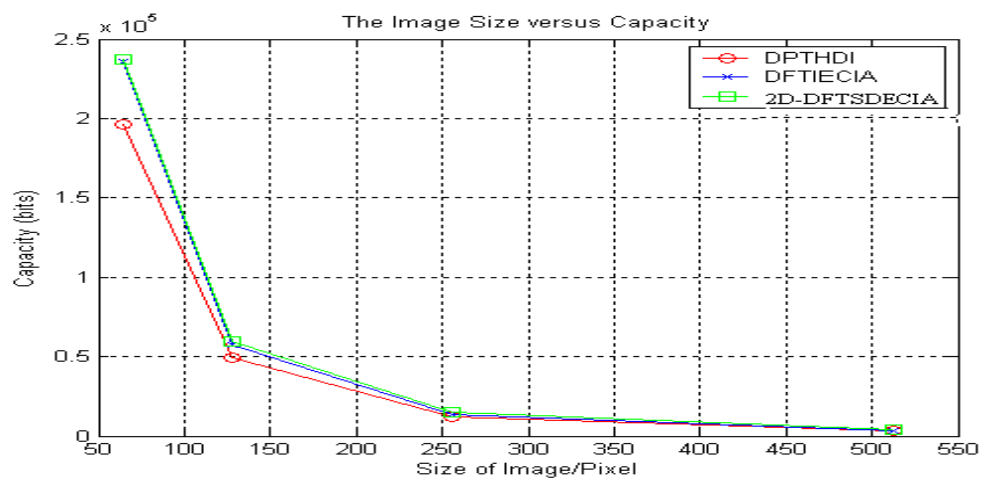

Figure 4. Comparisons Results of Size of Image/Pixel and Capacity (bytes) among Varying Sizes of Embedded 'Lena' Image with schemes DPTHDI [7], DFTIECIA [8] and 2D-DFTSDECIA

In Fig-5, the histogram analysis of Lena image is shown before and after embedding secret information in a individual channel wise manner. 
Signal \& Image Processing : An International Journal (SIPIJ) Vol.3, No.6, December 2012

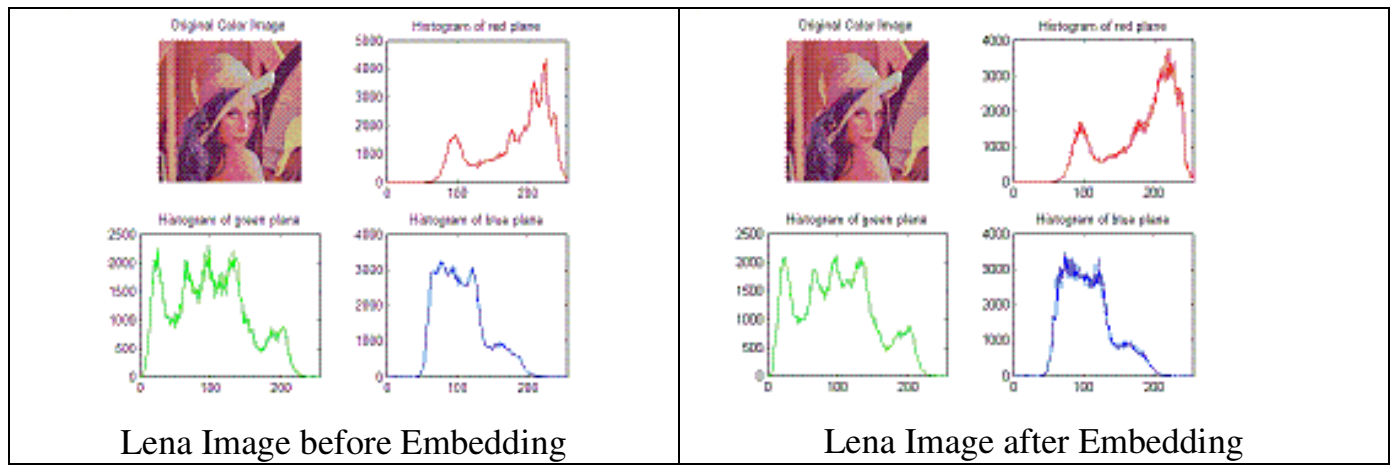

Figure 5. Comparisons Results of Histograms between of Original and Embedded Image of Lena

The histogram analysis shows the comparison results in terms of mean, standard deviation and median between original and embedded 'Lena' image in a channel wise manner. The experimental result in Table 3 also ensures that the difference between two images is very minimal and tough to detect for the attacker.

Table 3. Comparison results of Mean, Median and Standard Deviation of Original and Embedded Lena Image

\begin{tabular}{|c|c|c|c|c|}
\hline Image & Channel & Mean & Median & Standard Deviation \\
\hline \multirow{3}{*}{ Original Lena } & $\mathrm{R}$ & 180.22 & 197 & 49.05 \\
\cline { 2 - 5 } & $\mathrm{G}$ & 99.05 & 97 & 52.88 \\
\cline { 2 - 5 } & $\mathrm{B}$ & 105.41 & 100 & 34.06 \\
\hline \multirow{3}{*}{ Embedded Lena } & $\mathrm{R}$ & 180.13 & 196 & 49.09 \\
\cline { 2 - 5 } & $\mathrm{G}$ & 98.86 & 97 & 52.88 \\
\cline { 2 - 5 } & $\mathrm{B}$ & 105.44 & 101 & 34.29 \\
\hline
\end{tabular}

\section{Conclusion}

The 2D-DFTSDECIA scheme is an image authentication process in frequency domain to enhance the security compared to the existing algorithms. Authentication is done by embedding secret data in a carrier image. Using the technique, we can embed 29 bits in each 2 x 2 image block. Experimental results conform that the proposed algorithm achieves better experimental results than the DPTHDI [7] scheme and significantly improves the qualities and payload capacities over our previously proposed DFTIECIA [8] scheme.

\section{REFERENCES}

[1] Zhang, T., Ping, X.: A Fast and Effective Steganalytic Technique against JSteglike Algorithms. In: Proc. 8th ACM Symp. Applied Computing. ACM Press, New York (2003)

[2] J. Cox, J. Kilian, F. T. Leighton, and T. Shamoon, Secure spread spectrum watermarking for images, audio and video, in Proc. IEEE Int. Conf. Image Processing, Lausanne, Switzerland, Sep. 16-19, vol. 111, pp. 243-246, 1996.

[3] J. Cox, J. Kilian, F. T. Leighton, and T. Shamoon, Secure spread spectrum watermarking for multimedia, IEEE Trans. Image Processing, vol. 6, no. 12, pp. 1673-1687, 1997.

[4] N. Ahmidi, R. Safabkhsh, A novel DCT-based approach for secure color image watermarking, in Proc. Int. Conf. Information technology: Coding and Computing, vol. 2, pp. 709-713, Apr. 2004.

[5] H. Chou, Y. C. Li, A perceptually tuned subband image coder based on the measure of just-noticeable distortion profile, IEEE Trans. Circuits Syst. Video Technology vol. 5, no. 6, pp. 467-476, Dec. 1995.

[6] Allan G. Weber, The USC-SIPI Image Database: Version 5, Original release: October 1997, Signal and Image Processing Institute, University of Southern California, Department of Electrical Engineering. http://sipi.usc.edu/database/ (accessed on 25th January, 2010). 
Signal \& Image Processing : An International Journal (SIPIJ) Vol.3, No.6, December 2012

[7] Varsaki Eleni E., Fotopoulos Vassilis E., Skodras Athanassios N., "On the use of the discrete Pascal transform in hiding data in images", "Optics, Photonics, and Digital Technologies for Multimedia Applications”, Proc. of SPIE Vol. 7723, 77230L • (C) 2010 SPIE • CCC code: 0277-786X/10/\$18 • doi: 10.1117/12.854220, 2010.

[8] Mandal J.K, Ghosal, S.K, "A Novel DFT Based Information Embedding for Color Image Authentication (DFTIECIA)", Third International Conference on Communication Security and Information Assurance (CSIA 2012), Advances in Intelligent and Soft Computing, 2012, Springer, Volume 167/2012, 763-772, DOI: 10.1007/978-3-642-30111-7_73, May, 2012, Delhi.

\section{Authors}

Jyotsna Kumar Mandal, M. Tech.(Computer Science, University of Calcutta),Ph.D.(Engg., Jadavpur University) in the field of Data Compression and Error Correction Techniques, Professor in Computer Science and Engineering, University of Kalyani, India. Life Member of Computer Society of India since 1992 and life member of cryptology Research Society of India. Ex-Dean, Faculty of Engineering, Teachnology \& Management, working in the field of Network Security, Steganography, Remote Sensing \& GIS Application, Image Processing. 25 years of teaching and research experiences. Eight Scholars awarded Ph.D. one submitted and eight are pursuing. Total number of

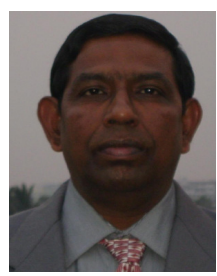
publications is more than two hundred thirty in addition of publication of five books from LAP Lambert, Germany.

Sudipta Kr Ghosal, is an Assistant Professor and Teacher in-charge of Computer Science \& Engineering department at Greater Kolkata College of Engineering \& Management, Kolkata. He received his bachelor of technology in Computer science and Engineering in 2007. He received his master of technology in IT (Courseware Engineering) from Jadavpur University, Kolkata, India in 2010. He is pursuing research for $\mathrm{PhD}$ in Color Image Authentication from University of Kalyani, India under the supervision of Prof. J.K.Mandal. Mr. Ghosal has around three years of experience in teaching and industry. He has eleven publications in the national and international conference proceedings and journals. 